\title{
Ecofisiologia da germinação dos genótipos de Theobroma cacao L.
}

\author{
Daniele Freisleben Lavanhole' ${ }^{1}$, Sara Dousseau Arantes ${ }^{1}$ [D, Lúcio de Oliveira Arantes' \\ Carlos Alberto Spaggiari Souza ${ }^{2}$ (D), Sheila Cristina Prucoli Posse' (1), \\ Basílio Cerri Neto' (1), Jeane Crasque' ${ }^{1}$ (1)
}

\author{
1 Instituto Capixaba de Pesquisa, Assistência Técnica e Extensão Rural, BR 101N, km 151, Linhares, Caixa postal 62, Espírito Santo, Brasil. \\ danieleflavanhole@hotmail.com \\ ${ }^{2}$ Comissão Executiva do Plano da Lavoura Cacaueira, BR 101, Km 150, Linhares, Espírito Santo, Brasil.
}

Recebido em 17.VI.2018

Aceito em 24.VI.2019

DOI 10.21826/2446-82312019v74e2019007

RESUMO - Objetivou-se com esse estudo avaliar a interação dos genótipos (Comum, TSH-1188, ESFIP-02, CCN-51, Ipiranga-01, SJ-02, PH16 , PS-1319, CEPEC-2002 e Catongo) com temperaturas $\left(15{ }^{\circ} \mathrm{C}, 2{ }^{\circ} \mathrm{C}, 25^{\circ} \mathrm{C}, 30^{\circ} \mathrm{C}, 35^{\circ} \mathrm{C}, 40{ }^{\circ} \mathrm{C}, 15-25{ }^{\circ} \mathrm{C}\right.$ e $\left.20-30{ }^{\circ} \mathrm{C}\right)$ e períodos de secagem (zero, dois e quatro dias) na germinação e no desenvolvimento pós-germinativo do cacaueiro. O estudo da interação entre genótipo/temperatura foi fatorial $10 \times 8$, enquanto a tolerância à dessecação foi fatorial $10 \times 3$. Foi realizada análise de variância e as médias foram comparadas pelo teste de agrupamento Scott-Knott $(\mathrm{p}<0,05)$. Para o ensaio de influência de temperatura, as médias obtidas foram submetidas ao teste de regressão. A resposta à temperatura foi influenciada de maneira distinta pelo genótipo. O genótipo TSH-1188 foi o único a apresentar comportamento bastante distinto, com germinabilidade e vigor acima das temperaturas testadas. Quanto à dessecação das sementes os genótipos avaliados tiveram redução significativa de vigor.

Palavras-chave: dessecação, porta-enxerto, sementes, temperatura

\begin{abstract}
Germination ecophysiology of germination of Theobroma cacao L. genotypes. The aim of this study was to evaluate the interaction of the genotypes with common temperatures (common, TSH-1188, ESFIP-02, CCN-51, Ipiranga-01, SJ-02, PH-16, PS-1319, CEPEC-2002 and Catongo) $\left(15^{\circ} \mathrm{C}, 20^{\circ} \mathrm{C}, 25^{\circ} \mathrm{C}, 30^{\circ} \mathrm{C}, 35^{\circ} \mathrm{C}, 40^{\circ} \mathrm{C}, 15-25^{\circ} \mathrm{C}\right.$ and $\left.20-30{ }^{\circ} \mathrm{C}\right)$ and drying periods $(0,2$ and 4 days $)$ on germination and post-germination cacao tree. The genotype / temperature interaction study was a $10 \times 8$ factorial, while the desiccation tolerance was a $10 \times 3$ factorial. Variance analysis was performed and the means were compared using the Scott-Knott grouping test $(p<0.05)$. For the temperature influence test, the means obtained were submitted to the regression test. The temperature response was influenced differently by the genotype. The TSH-1188 genotype was the only one to present quite distinct behavior, with germinability and vigor above the temperatures tested. As for seed desiccation, the evaluated genotypes had a significant reduction of vigor.
\end{abstract}

Keywords: desiccation, rootstocks, seeds, temperature

\section{INTRODUÇÃO}

O Brasil é o sétimo maior produtor mundial de cacau, com uma área colhida de 704.122 ha, produção de $273.793 \mathrm{t}$ e rendimento de $3.888 \mathrm{hg} / \mathrm{ha}^{-1}$, segundo a Food and Agriculture Organization (FAO 2017). A maior parte das lavouras cacaueiras foi implantada com mudas advindas de sementes, no entanto, atualmente a propagação vegetativa por enxertia é a mais indicada para implantação das novas lavouras visando a resistência às doenças e ao aumento da produtividade. A propagação do porta-enxerto é predominantemente via seminal, sendo a germinação de sementes etapa crucial que se relaciona diretamente com o custo de produção das mudas (Venial et al. 2017).

Os genótipos TSH-1188 e Comum atualmente são os mais empregados no Espírito Santo como porta-enxertos e apresentam características distintas em relação ao desenvolvimento. O TSH-1188 é um híbrido descendente do grupo Forastero e Trinitário, que apresenta elevado vigor vegetativo e tolerância a Ceratocystis cacaofunesta Engelbr. \& T.C. Harr. (Engelbrecht \& Harrington 2005) e Moniliophthora perniciosa Aime \& Phillips-Mora (Aime \& Phillips-Mora 2005) ao estresse hídrico. No entanto, apresenta segregação quanto ao desenvolvimento vegetativo, o que acarreta em elevada desuniformidade no tamanho das mudas, dificultando a obtenção de plantas uniformes para se efetuar a enxertia (Gomes \& Sodré 2015). Esta desuniformidade exige que o viveirista efetue maior semeadura para obtenção de plantas com o padrão exigido para a enxertia. Por outro lado, o genótipo Comum, por ser uma variedade do grupo Forastero é bastante uniforme, no entanto, possui desenvolvimento mais lento, demandando um período maior de enviveiramento para atingir o padrão de enxertia (Arévalo et al. 2012).

No entanto, é fundamental a ampliação da base genética na obtenção do porta-enxerto, para reduzirem-se as probabilidades de perdas em decorrência do surgimento de fatores bióticos e abióticos estressantes advindos das 
mudanças climáticas. Sendo assim, a identificação de genótipos com maior plasticidade fenotípica às diversas condições edafoclimáticas, são de grande interesse pelo setor produtivo de mudas (Schmitz et al. 2014; Madeira et al. 2016). Desta forma, consistindo a propagação dos portaenxertos para cacaueiros predominantemente via seminal, o conhecimento do processo germinativo é fundamental, pois diversos fatores externos (ambientais) e internos (dormência, inibidores e promotores da germinação) interferem nesse processo.

A germinação das sementes é um processo fisiológico complexo que inicia com a embebição de água e culmina na protrusão radicular, sendo a etapa seguinte denominada desenvolvimento pós-germinativo, caracterizada pelo crescimento e desenvolvimento das plântulas (Dousseau et al. 2013). O processo germinativo é regulado por vários fatores ambientais, como a umidade, oxigênio, temperatura, luz e nutrientes (Penfield et al. 2005, Socolowski et al. 2008, Seo et al. 2009). A taxa inicial de embebição e a temperatura podem alterar acentuadamente a germinação e o vigor das sementes (Ataíde et al., 2016). A resposta aos fatores ambientais é dependente tanto da adaptação da espécie em função das características do seu centro de origem (Parent \& Tardieu 2012), quanto da sua plasticidade fenotípica.

A maioria das espécies de regiões tropicais germina em uma ampla faixa de temperatura $\left(15-35^{\circ} \mathrm{C}\right)$, estando à temperatura mínima (infraótima) entre $10^{\circ} \mathrm{C} \mathrm{e} 15^{\circ} \mathrm{C}$ e a máxima (supraótima) entre $35^{\circ} \mathrm{C} \mathrm{e} 40^{\circ} \mathrm{C}$ (Amri 2011). No entanto, existe uma faixa de temperatura considerada ótima, na qual a germinabilidade e o vigor são máximos, acima e abaixo da qual ocorre diminuição (Custódio et al. 2009; Lamarca et al. 2013). A capacidade de uma espécie de se estabelecer em um ambiente natural ou agrícola é definida pela adaptação aos extremos de temperatura, bem como pela sua plasticidade fenotípica a este fator ambiental. Portanto, a regeneração das populações a partir de sementes é definida pela resposta a temperatura (Dürr et al. 2015). Estudos sobre a temperatura ideal para germinação de genótipos do cacaueiro são escassos, sendo relevante caracterizar a temperatura adequada nos genótipos utilizados como porta-enxerto.

O teor de umidade nas sementes é outro fator que influencia decisivamente no processo germinativo e na sua longevidade (Dousseau et al. 2011). As sementes de cacaueiro são classificadas como recalcitrantes, sendo muito sensível a dessecação, o que se torna um obstáculo para a conservação, devido à rápida perda de viabilidade (Changrun \& Wendell 1999). Segundo Chandel et al. (1995), sementes e eixos embrionários submetidos à secagem rápida ( $4 \mathrm{~h}$ em sílica gel) perderam totalmente a viabilidade e apresentaram alteração na ultraestrutura (nucléolos, mitocôndria, retículo endoplasmático, complexo de Golgi, ribossomos), aumento na condutividade hidráulica, na peroxidação lipídica e nos teores de carboidratos solúveis, indicando haver perda da integridade da membrana e degradação do amido. De acordo com Changrun \& Wendell
(1999), a sensibilidade à dessecação varia em função do estádio de maturação da semente, ocorrendo um aumento progressivo na peroxidação lipídica durante a dessecação e uma diminuição na proteção enzimática.

Além de poucos estudos sobre a germinação de cacaueiro, os existentes são de 1986 (Figueiredo 1986a, b), destacando-se a importância de se caracterizar o processo germinativo dos principais genótipos cultivados na região norte do Espírito Santo. Desta forma, objetivou-se com esse trabalho avaliar a interação do genótipo com temperaturas e períodos de secagem na germinação e no desenvolvimento pós-germinativo do cacaueiro.

\section{MATERIAL E MÉTODOS}

Foram avaliados os principais genótipos existentes na Comissão Executiva do Plano da Lavoura Cacaueira (CEPLAC, 2017) do município de Linhares, Espírito Santos (Comum, TSH-1188, ESFIP-02, CCN-51, Ipiranga-01, SJ-02, PH-16, PS-1319, CEPEC-2002 e Catongo), sendo os frutos coletados no ponto de maturação aparente (casca amarelada ou alaranjada), de plantas cultivadas no campo de manutenção de genótipos da Comissão Executiva do Plano da Lavoura Cacaueira (CEPLAC, 2017), localizada no município de Linhares, Espírito Santo. A coleta dos frutos foi feita respeitando o ponto de maturação de cada genótipo e em uma única planta na matriz.

O município de Linhares está localizado a uma latitude Sul de 19'23'59" e uma longitude Oeste de Greenwich de $40^{\circ} 04^{\prime} 01^{\prime \prime} \mathrm{W}$, a $28 \mathrm{~m}$ acima do nível do mar. Segundo a classificação climática proposta por Köppen, o município é caracterizado como Aw, ou seja, tropical úmido, com inverno seco e chuvas máximas no verão, dada pela temperatura média do mês mais frio inferior a $18^{\circ} \mathrm{C}$ e a precipitação do mês mais seco inferior a $60 \mathrm{~mm}$. As médias anuais de precipitação foram $1277 \mathrm{~mm}$, enquanto que a evapotranspiração potencial anual é de $1224 \mathrm{~mm}$ e a deficiência hídrica $69 \mathrm{~mm}$ (Alvares et al. 2013).

Imediatamente após a coleta dos frutos, a mucilagem foi retirada por meio da fricção com as mãos em pó de serra seco (Corrêa et al. 2011) e levadas ao Laboratório de Sementes do Instituto Capixaba de Pesquisa, Assistência Técnica e Extensão Rural (INCAPER), onde foram lavadas em água corrente com auxílio de uma peneira e imersas em solução Captan a $1 \%(\mathrm{p} / \mathrm{v})$, por 15 minutos para desinfestação. Foram instalados dois ensaios, sendo um para avaliar a influência da temperatura e outro para a tolerância à dessecação.

As temperaturas avaliadas foram $15^{\circ} \mathrm{C}, 20^{\circ} \mathrm{C}, 25^{\circ} \mathrm{C}$, $30{ }^{\circ} \mathrm{C}, 35^{\circ} \mathrm{C}$ e $40{ }^{\circ} \mathrm{C}$ constantes e $15-25^{\circ} \mathrm{C}$ e $20-30{ }^{\circ} \mathrm{C}$ alternadas, em sistema de rolo utilizando dois papéis Germitest, em germinador tipo Biochemical Oxigen Demand (B.O.D), com 58\% de UR, em fotoperíodo de 12 horas.

No ensaio de tolerância à dessecação, o grau de umidade foi determinado em base úmida, pelo método da estufa a $105^{\circ} \mathrm{C} \pm 3^{\circ} \mathrm{C}$ por 24 horas (Brasil 2009), utilizando-se três 
repetições de $10 \mathrm{~g}$ de sementes. Após, a retirada da umidade superficial, as sementes foram mantidas sob bancada para secagem lenta, sendo avaliadas durante zero, dois e quatro dias. Foram realizadas avaliações quanto ao teor de umidade $(\% \mathrm{U})$ expresso em base úmida de acordo com as Regras para análise de sementes-RAS (Brasil, 2009). Posterior ao período de secagem as sementes foram colocadas em B.O.D a $30^{\circ} \mathrm{C}$ para a germinação.

Foram realizadas avaliações diárias da protrusão da radícula e calculadas a germinabilidade e índice de velocidade de germinação (IVG), segundo Maguire (1962). Após sete dias efetuou-se a análise de plântulas normais, as quais foram sendo classificadas de acordo com o seu desenvolvimento em Plântulas Normais Vigorosas e Fracas.

Os ensaios foram conduzidos em delineamento experimental inteiramente casualizado com quatro repetições de 25 sementes, em esquema fatorial. $\mathrm{O}$ estudo da interação entre genótipo e temperatura foi fatorial 10 (genótipos) x 8 (temperaturas) $\left(15^{\circ} \mathrm{C}, 20^{\circ} \mathrm{C}, 25^{\circ} \mathrm{C}, 30^{\circ} \mathrm{C}\right.$, $35^{\circ} \mathrm{C}$ e $40^{\circ} \mathrm{C}$ constantes e $15-25^{\circ} \mathrm{C}$ e $20-30^{\circ} \mathrm{C}$ alternadas), enquanto o ensaio de tolerância à dessecação foi fatorial 10 (genótipos) x 3 (períodos de secagem: 0,2 e 4 dias). A análise estatística foi realizada mediante o uso do programa estatístico Sisvar (Ferreira 2014). Foi realizada a análise de variância e as médias foram comparadas pelo teste de agrupamento Scott-Knott $(\mathrm{p}<0,05)$, a $5 \%$ de probabilidade para ambos os ensaios (influência da temperatura e tolerância à dessecação). Para o ensaio de influência de temperatura, as médias obtidas foram submetidas ao teste de regressão e avaliado o ajuste ao modelo polinomial, utilizando-se o teste F a 5\% de probabilidade e o coeficiente de determinação. Quando a resposta não se ajustou aos modelos propostos, procedeu-se a comparação das médias pelo teste de ScottKnott $(\mathrm{p}<0,05)$. Os pontos de máximo foram calculados com base nas equações estabelecidas.

Em ambos os ensaios foi utilizado o método de Singh (1981) para analisar os caracteres que mais contribuíram para a divergência genética entre os genótipos avaliados, por meio da distância generalizada de Mahalanobis $\left(\mathrm{D}^{2}\right)$. Utilizaram-se as análises de agrupamento e variáveis canônicas para a indicação dos melhores genótipos. Estas análises estatísticas foram realizadas utilizando-se o Programa Genes (Cruz, 2006).

\section{RESULTADOS E DISCUSSÃO}

Os genótipos de cacaueiro (Comum, TSH-1188, ESFIP-02, CCN-51, Ipiranga-01, SJ-02, PH-16, PS-1319, CEPEC-2002 e Catongo) apresentaram germinabilidade máxima na faixa de temperatura entre $24^{\circ} \mathrm{C}$ e $32{ }^{\circ} \mathrm{C}$, contudo, a velocidade de germinação foi superior entre $32 \mathrm{C} \mathrm{e} 40^{\circ} \mathrm{C}$. Existem diversos trabalhos que comprovam que temperaturas elevadas proporcionam uma germinação mais rápida, contudo, a porcentagem é reduzida (Ferraz et al. 2012, Lloh et al. 2014, Holanda et al. 2015). O requerimento de temperatura para a formação da plântula foi mais restrito, variando apenas entre $28^{\circ} \mathrm{C}$ e $29^{\circ} \mathrm{C}$ para os genótipos estudados (Fig. 1).

Considerando que o conceito de temperatura ideal é aquela onde se tem a máxima germinação no menor tempo (Marcos-Filho 2015), não é possível definir uma única temperatura ótima para a espécie de cacaueiro, visto que, os genótipos apresentaram distintas respostas quanto às temperaturas estudadas (Fig. 1). No entanto, ao se analisar o resultado do teste de agrupamento por meio dos componentes principais (Fig. 2), pode-se recomendar a temperatura de $30^{\circ} \mathrm{C}$ para a condução dos testes de germinação do cacaueiro, uma vez que praticamente todos os genótipos apresentaram respostas semelhantes constituindo assim, um grupo, considerando o índice de velocidade de germinação e a porcentagem de plântulas normais vigorosas. Exceto o TSH-1188 que apresentou comportamento bastante distinto, com germinabilidade e vigor acima das temperaturas testadas. Figueiredo (1986b), não descreveu o genótipo de cacaueiro estudado, mas também considerou a temperatura de $30^{\circ} \mathrm{C}$ ideal, uma vez que proporcionou o número máximo de plântulas no menor período de tempo. A $35{ }^{\circ} \mathrm{C}$ Figueiredo (1986b) também observou maior velocidade na emissão do eixo embrionário, contudo, a formação da plântula foi prejudicada.

Através do teste de agrupamento por meio dos componentes principais (Fig. 2), foi possível verificar que as variáveis índice de velocidade de emergência e plântulas normais vigorosas foram as que mais explicaram as respostas à temperatura. A variável germinabilidade não representa os tratamentos, pois na maioria das sementes ocorreu a protrusão radicular. Sendo, portanto, recomendado que os ensaios que avaliem a resposta à temperatura considerem estas variáveis para a melhor interpretação dos resultados. Além disso, em ensaios que abordem a interação entre genótipos e temperaturas, deve ser priorizada a análise multivariada para a obtenção de resultados mais precisos, ao invés de utilizar os testes de médias comumente empregados.

Os genótipos de cacaueiro apresentaram tolerância distinta quanto aos extremos térmicos avaliados, sendo o TSH-1188 o único que manteve a germinabilidade próximo a $100 \%$ e o vigor elevado em todas as temperaturas avaliadas (Fig. 1). O genótipo CEPEC-2002 apresentou a menor tolerância aos extremos térmicos, sendo tanto a germinabilidade quanto o vigor reduzidos nas temperaturas de $15^{\circ} \mathrm{C}, 20^{\circ} \mathrm{C}, 40^{\circ} \mathrm{C}$ e $15-25^{\circ} \mathrm{C}$. A temperatura de $15^{\circ} \mathrm{C}$ também promoveu redução severa no processo germinativo dos genótipos SJ-02 e Catongo. O genótipo PH-16 também teve a germinabilidade e o vigor bastante reduzidos na temperatura de $40^{\circ} \mathrm{C}$. Segundo Figueiredo (1986b), a faixa ideal de temperatura para germinação de cacaueiro está entre 18 a $30^{\circ} \mathrm{C}$ e seu limite inferior de temperatura letal é de $10^{\circ} \mathrm{C}$. No entanto, no presente ensaio, podemos concluir que esta sensibilidade aos extremos térmicos varia em função do genótipo. 


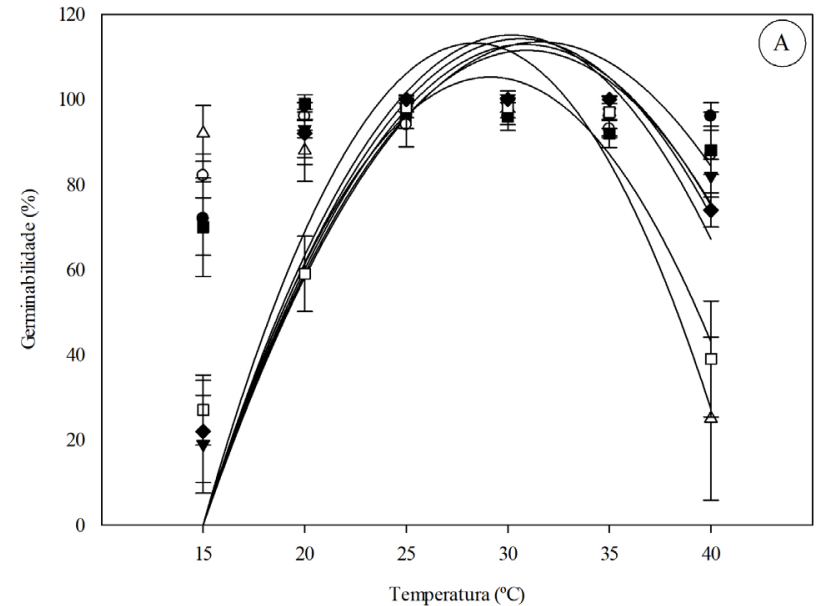

- Comum $y=9,771429+5,648571 x-0,088571 x^{2} \quad r^{2}=91$

- $C C N-51 \mathrm{y}=37,742857+4,188571 \mathrm{x}-0,074286 \mathrm{x}^{2} \quad \mathrm{r}^{2}=84$

> SJ-02 $\mathrm{y}=-203,021429+20,619286 \mathrm{x}-0,340714 \mathrm{x}^{2} \quad \mathrm{r}^{2}=87$

$\triangle \quad$ PH-16 y=-57,657143+13,477143x-0,277143x $x^{2} \quad r^{2}=76$

- PS-1319 y=-4,792857+7,185000x-0,123571 $x^{2} \quad \mathrm{r}^{2}=74$

ㅁ CEPEC $-2002 \mathrm{y}=-255,414286+24,958571 \mathrm{x}-0,435714 \mathrm{x}^{2} \quad \mathrm{r}^{2}=93$

- Catongo $y=-213,2000+21,737143 x-0,365714 x^{2} \quad r^{2}=90$

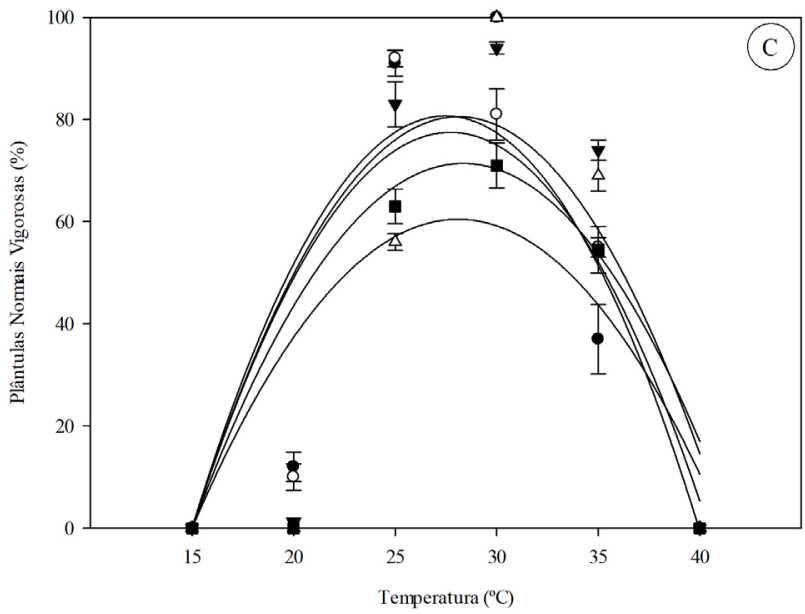

- TSH-1188 y=-370,021429+32,419286x-0,580714 $x^{2} \quad \mathrm{r}^{2}=78$

- ESFIP-02 $y=-349,307143+30,447857 x-0,540714 x^{2} \quad r^{2}=81$

v CCN-51 y $=-376,321429+32,075000 x-0,559286 x^{2} \quad r^{2}=76$

$\triangle \quad$ SJ-02 y $=-340,192857+28,659286 x-0,495000 x^{2} \quad r^{2}=70$

- $\mathrm{PH}-16 \mathrm{y}=-283,753571+24,178214 \mathrm{x}-0,421786 \mathrm{x}^{2} \quad \mathrm{r}^{2}=75$

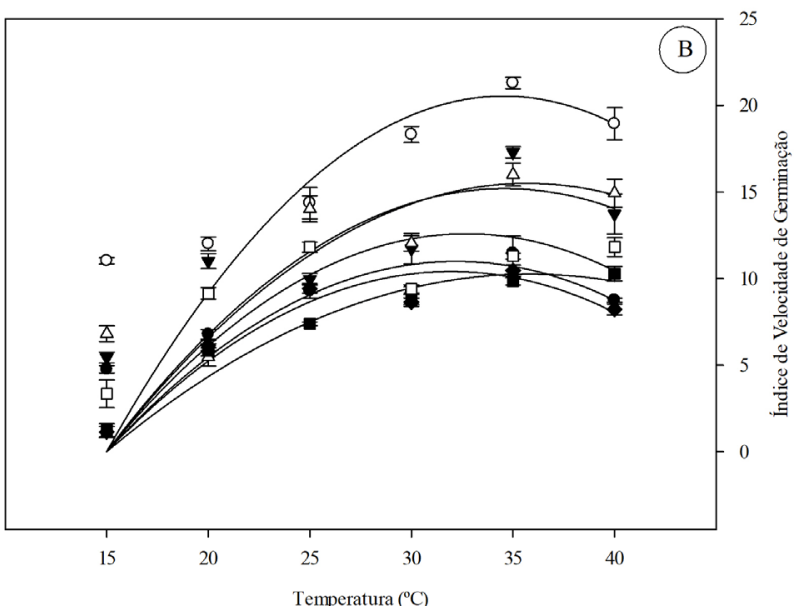

- Comum $y=-8,977652+1,161247 x-0,017587 x^{2} \quad r^{2}=88$

- TSH- $1188 \mathrm{y}=-2,136943+0,963838 \mathrm{x}-0,010108 \mathrm{x}^{2} \quad \mathrm{r}^{2}=88$

$\nabla \quad$ ESFIP-02 $y=-7,609937+1,109151 \mathrm{x}-0,013712 \mathrm{x}^{2} \quad \mathrm{r}^{2}=76$

$\Delta \quad$ Ipiranga-01 $\mathrm{y}=-7,750827+1066301 \mathrm{x}-0,012092 \mathrm{x}^{2} \mathrm{r}^{2}=76$

- SJ-02 $\mathrm{y}=-13,117821+1,232164 \mathrm{x}-0,016316 \mathrm{x}^{2} \quad \mathrm{r}^{2}=98$

ㅁ $\quad$ PS-1319 $\mathrm{y}=-12,191736+1,421944 \mathrm{x}-0,021039 \mathrm{x}^{2} \quad \mathrm{r}^{2}=80$

- Catongo $y=-20,672691+1,923822 x-0,030028 x^{2} \quad r^{2}=95$

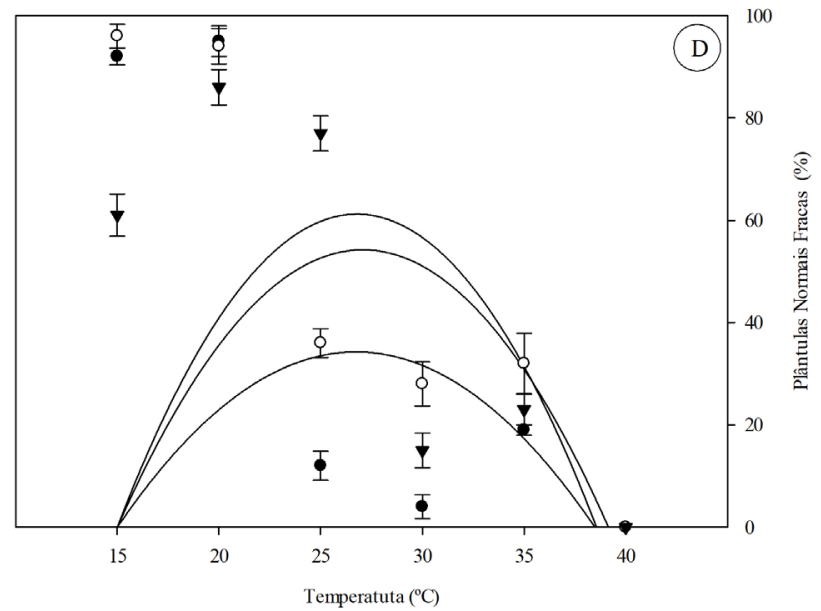

- $\mathrm{CCN}-51 \mathrm{y}=284,014286-15,055714 \mathrm{x}+0,021429 \mathrm{x} 2 \quad \mathrm{r}^{2}=80$

- PH-16 y=201,414286-7,701429x+0,070000x $\quad \mathrm{r}^{2}=88$

$\checkmark$ CEPEC-2002 $\mathrm{y}=47,08+3,580000 \mathrm{x}-0,122857 \mathrm{x}^{2} \quad \mathrm{r}^{2}=75$

Figs. 1A-D. Comportamento germinativo e pós-germinativo de sementes de dez genótipos de cacaueiro, submetidos a temperaturas constantes (15 ${ }^{\circ} \mathrm{C}, 20^{\circ} \mathrm{C}, 25^{\circ} \mathrm{C}, 30^{\circ} \mathrm{C}, 35^{\circ} \mathrm{C}$ e $40^{\circ} \mathrm{C}$ ) no município de Linhares-ES. A. Germinabilidade; B. Índice de velocidade de germinação; C. Plântulas normais vigorosas; D. Plântulas normais fracas.

A formação da plântula do cacaueiro foi severamente limitada nos extremos térmicos avaliados, sendo nula a porcentagem de plântulas normais vigorosas para todos os genótipos a $15^{\circ} \mathrm{C}, 40^{\circ} \mathrm{C}$ e $15-25^{\circ} \mathrm{C}$ (Fig. 1). O genótipo SJ02 foi o mais sensível à baixa temperatura, sendo observada elevada inibição na formação da plântula a $15^{\circ} \mathrm{C}$, chegando a $33 \%$ de plântulas normais fracas. Na temperatura de $20^{\circ} \mathrm{C}$ apenas o TSH-1188 e o ESFIP-02 formaram plântulas normais vigorosas, sendo respectivamente $12 \%$ e $10 \%$. A temperatura de $40^{\circ} \mathrm{C}$ inibiu totalmente a formação da plântula, embora tenha ocorrido protrusão radicular, ocorreu logo em seguida à morte. Contudo, nas temperaturas de $15^{\circ} \mathrm{C}, 20^{\circ} \mathrm{C}$ e $15-25^{\circ} \mathrm{C}$ a maioria dos genótipos manteve elevada porcentagem de plântulas normais fracas, sendo, portanto, um indicativo de inibição no desenvolvimento das estruturas da plântula, principalmente considerando os pelos radiculares, pois os mesmos foram um dos critérios utilizados na sua classificação. Segundo Pereira et al. (2001), em baixas temperaturas a plântula deixa de investir no sistema radicular para investir no desenvolvimento da 

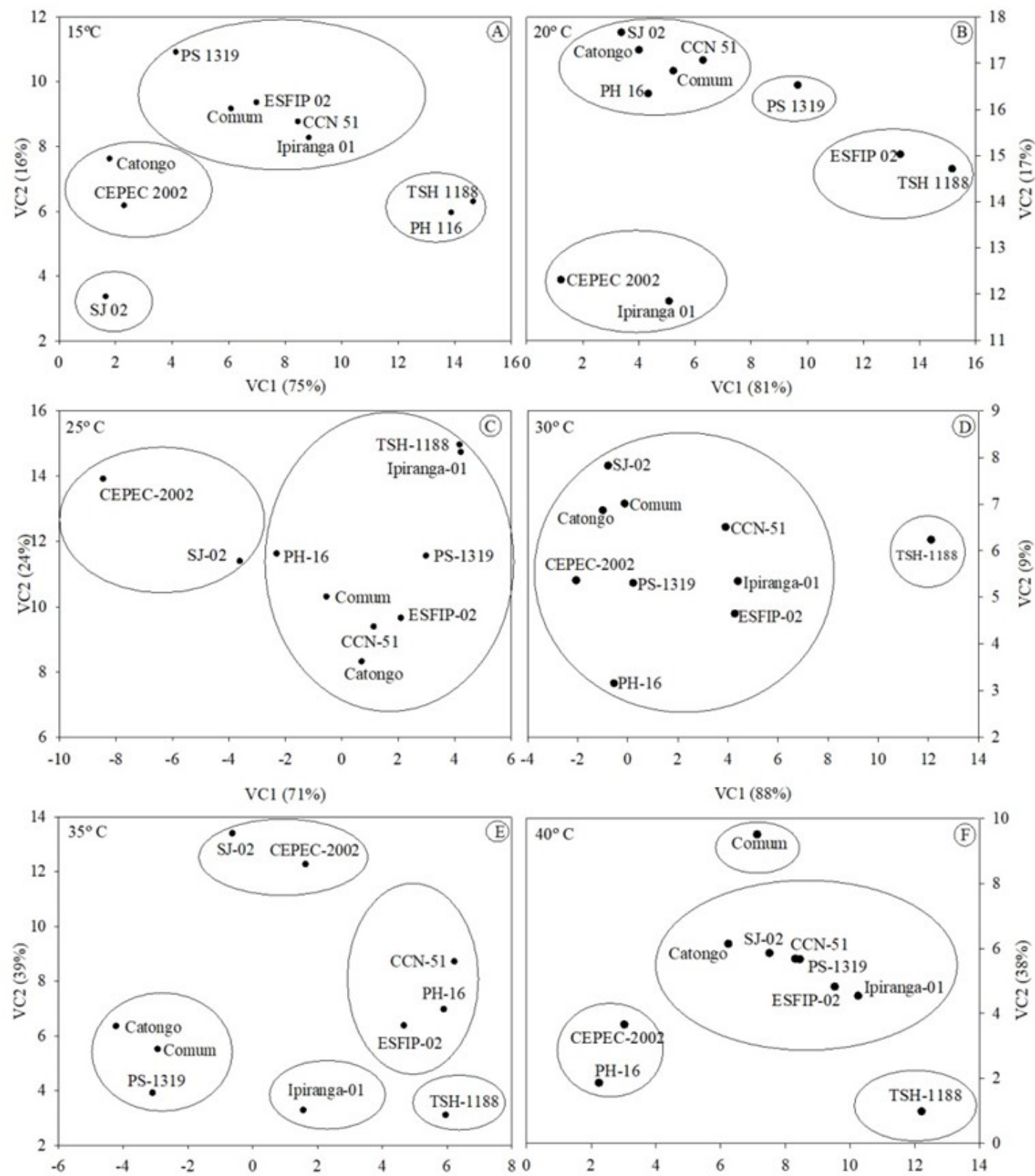

$\operatorname{VCl}(52 \%)$
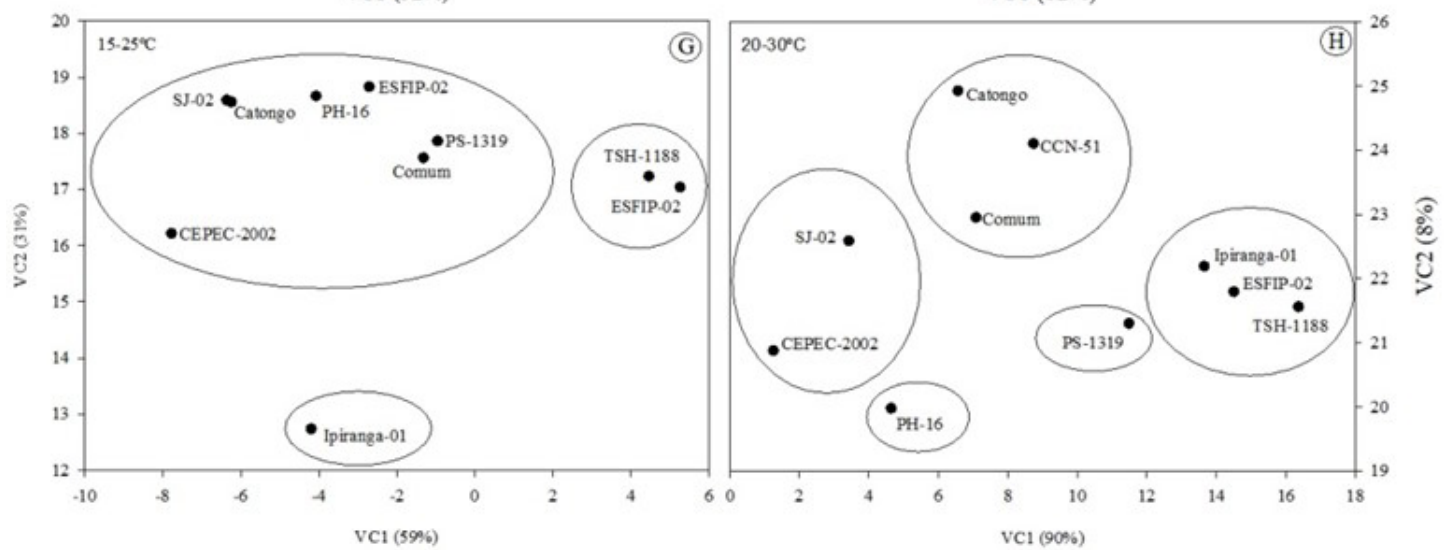

Figs. 2A-H. Dispersão de dez genótipos de cacaueiro da Comissão Executiva do Plano da Lavoura Cacaueira no município de Linhares/ES, usando as coordenadas obtidas da matriz de distância generalizada de Mahalanobis $\left(\mathrm{D}^{2}\right)$, pelo procedimento estatístico baseado na minimização das diferenças entre as distâncias originais e aquelas no gráfico. Os agrupamentos se estabeleceram de acordo com a temperatura, sendo: A. $15^{\circ} \mathrm{C}$; B. $20{ }^{\circ} \mathrm{C}$; C. 25 ${ }^{\circ} \mathrm{C}$; D. $30^{\circ} \mathrm{C}$; E. $35^{\circ} \mathrm{C}$; F. $40{ }^{\circ} \mathrm{C}$; G. $15-25^{\circ} \mathrm{C}$; H. $20-30{ }^{\circ} \mathrm{C}$. 
parte aérea realizando a translocação de compostos para a manutenção da integridade do seu sistema. Figueiredo (1986b) relata que a $35^{\circ} \mathrm{C}$ a raiz primária e o hipocótilo do cacaueiro se tornam escurecidos e necrosados, ao contrário do que observamos neste ensaio.

No presente ensaio pode-se verificar que a faixa de temperatura foi mais restrita para a formação da plântula do que para o processo germinativo. Este resultado pode ser explicado pelo fato do desenvolvimento pósgerminativo ser considerado mais complexo que a germinação, pois a formação da plântula é dependente de uma maior quantidade de processos fisiológicos, como os tropismos, a fotomorfogênese, expressão de pelos radiculares, crescimento da raiz e da parte aérea. Além disto, é possível inferir que as características do cacaueiro quanto ao desenvolvimento da semente também influenciaram nesta resposta. Ou seja, como o embrião já está bem desenvolvido na dispersão da semente e o teor de umidade é elevado, o beneficiamento com a retirada da polpa propicia a germinação imediata. Devido aos altos teores de umidade das sementes do cacaueiro, a embebição é lenta e não caracteriza padrão-trifásico (Venial et al. 2017).

No caso das sementes do cacaueiro, o eixo embrionário é bastante desenvolvido, sendo relativamente menor a quantidade necessária de esqueletos de carbono e energia, para que ocorra a protrusão radicular, comparativamente com a formação da plântula. Provavelmente a energia e o carbono necessários advêm da mobilização da sacarose, que segundo Afoakwa et al. (2013) constitui 90\% dos carboidratos armazenados. Sendo assim, pode-se considerar que a resposta à temperatura na protrusão radicular das sementes de cacaueiro é mais relacionada com a atividade enzimática e integridade da membrana plasmática, do que com a velocidade de embebição.

A má formação das plântulas pode ser justificada pelo efeito da temperatura na viscosidade do citosol, fluidez e integridade de membrana, atividade antioxidante e taxas de transcrição e tradução (Long et al. 2014). O estresse ocasionado por alta temperatura gera um aumento no nível de peroxidação lipídica e peróxido de hidrogênio. As enzimas antioxidantes podem desempenhar um papel importante na termotolerância por eliminação de radicais livres para proteger a peroxidação lipídica e, consequentemente, melhorar a termostase da membrana celular (Ali et al. 2013). Flores et al. (2014), verificaram que as atividades das enzimas antioxidantes superóxido dismutase e catalase apresentaram alterações ao longo da germinação em $15^{\circ} \mathrm{C}$ e $40{ }^{\circ} \mathrm{C}$, demonstrando que nestas temperaturas ocorrem danos oxidativos.

Na faixa infraótima ocorre à imobilização gradual dos compostos de reserva, reduzindo gradativamente a porcentagem de germinação (Lopes \& Franke 2011), em função principalmente da redução na atividade das enzimas envolvidas no metabolismo (Rosental et al. 2014). Temperaturas baixas também interferem nas propriedades da membrana plasmática, reduzindo a capacidade seletiva, pela redução na sua fluidez (Mertz et al. 2009), portanto, um mecanismo de tolerância que pode ser ativado é a alteração da composição dos lipídeos de membrana. Em temperaturas mais baixas é observado aumento nos níveis de giberelina (GA) (Penfield et al. 2005, Toh et al. 2008), no entanto, até certo ponto, devido a redução na atividade das enzimas envolvidas na síntese hormonal. $\mathrm{O}$ desencadeamento de mecanismos associados ao estresse e o ABA pode levar ao aumento da síntese das moléculas antioxidantes, reduzindo a atividade da enzima catalase e consecutivamente melhorando o desempenho da germinação da semente (Lira et al. 2015).

Chandel et al. (1995) observou numerosos ribossomos no citoplasma das sementes de cacaueiro, indicando alta atividade metabólica. Muitas vezes estas sementes de cacaueiro já iniciam o desenvolvimento do eixo embrionário e até mesmo a protrusão radicular ainda dentro do fruto (germinação pós-colheita). A germinação pós-colheita é inibida pela presença da polpa (compostos inibidores e/ ou potencial osmótico elevado) (Figueiredo 1986c) e pela baixa relação ABA/GA. Esta germinação pós-colheita é comumente observada nos genótipos Catongo, Comum, PH-16 e CEPEC-2002.

O teor de umidade das sementes recém-colhidas dos genótipos de cacaueiro apresentou variação entre $49 \%$ e $58 \%$ (Tab. 1). O conteúdo de água em uma semente é uma característica variável em função da espécie, bem como do genótipo, sendo até certo ponto influenciada pelo ambiente. Está relacionada com a própria formação da semente, sendo muito influenciada pela sua composição química. No caso do cacaueiro, a composição química das sementes apresenta grande variação entre os genótipos sendo predominante lipo-proteicas, com conteúdo de lipídeos entre 19\% a 60\%, proteínas entre $10 \%$ a $30 \%$ e carboidratos entre 10 a $15 \%$ (Bucheli et al. 2001, Martini \& Tavares 2005).

Os resultados encontrados neste ensaio quanto ao teor de umidade foram diferentes do observado por Venial et al. (2017) estudando os genótipos CCN-51, PH-16, CEPEC-2002, Ipiranga-01, SJ-02, PS-1319, TSH-1188 e Comum, para os quais eles observaram valores entre $30 \% \mathrm{e}$ $43 \%$ de umidade nas sementes recém-colhidas. Esta variação tão grande de conteúdo de umidade provavelmente ocorreu devido aos procedimentos que estes autores utilizaram durante o beneficiamento das sementes, principalmente a etapa de desinfestação com álcool etílico a $70 \%$ por 1 minuto, que acarretou em desidratação parcial.

A redução no teor de umidade das sementes ao longo do período de secagem também foi variável entre os genótipos (Tab. 1). Após dois dias de secagem o teor de umidade variou entre $27 \%$ e $38 \%$, sendo observada redução variável entre $33 \%$ a $51 \%$ no conteúdo de umidade das sementes em relação às sementes recém-colhidas, sendo que o TSH1188 apresentou a menor redução e o Comum a maior. $\mathrm{O}$ teor de umidade após quatro dias de secagem variou entre 14 e $21 \%$, sendo observada redução acentuada para todos os genótipos, cujo valor variou entre $64 \%$ a $73 \%$ em relação às sementes recém-colhidas. No ensaio efetuado por Venial et al. (2017), a perda de umidade aos dois dias 
Tabela 1. Caracterização dos processos germinativo e pós-germinativo de sementes de dez genótipos de cacaueiro submetidas ao período de secagem (recém-colhidas, dois e quatro dias) no município de Linhares, Espírito Santo.

\begin{tabular}{|c|c|c|c|c|c|c|c|c|c|c|}
\hline & Comum & $\begin{array}{c}\text { TSH } \\
1188\end{array}$ & ESFIP-02 & CCN-51 & Ipiranga-01 & SJ-02 & PH-16 & PS-1319 & CEPEC-2002 & Catongo \\
\hline Dias & \multicolumn{10}{|c|}{ Umidade $(\%) \quad \mathrm{CV}=7 \%$} \\
\hline Recém-colhidas & $52 \mathrm{Ba}^{*}$ & $52 \mathrm{Ba}$ & $58 \mathrm{Aa}$ & $49 \mathrm{Ba}$ & $54 \mathrm{Ba}$ & $52 \mathrm{Ba}$ & $58 \mathrm{Aa}$ & $53 \mathrm{Ba}$ & $57 \mathrm{Aa}$ & $52 \mathrm{Ba}$ \\
\hline 2 & $27 \mathrm{Bb}$ & $35 \mathrm{Ab}$ & $35 \mathrm{Ab}$ & $28 \mathrm{Bb}$ & $31 \mathrm{Bb}$ & $28 \mathrm{Bb}$ & $38 \mathrm{Ab}$ & $33 \mathrm{Ab}$ & $28 \mathrm{Bb}$ & $28 \mathrm{Bb}$ \\
\hline 4 & $14 \mathrm{Bc}$ & $16 \mathrm{Bc}$ & $16 \mathrm{Bc}$ & $15 \mathrm{Bc}$ & $16 \mathrm{Bc}$ & $14 \mathrm{Bc}$ & $21 \mathrm{Ac}$ & $18 \mathrm{Ac}$ & $16 \mathrm{Bc}$ & $16 \mathrm{Bc}$ \\
\hline \multirow[t]{2}{*}{ CV (\%) } & 7 & & & & & & & & & \\
\hline & \multicolumn{9}{|c|}{ Germinabilidade (\%) } & \\
\hline Recém-colhidas & $100 \mathrm{Aa}$ & $100 \mathrm{Aa}$ & $97 \mathrm{Aa}$ & $100 \mathrm{Aa}$ & $100 \mathrm{Aa}$ & $100 \mathrm{Aa}$ & $100 \mathrm{Aa}$ & $96 \mathrm{Aa}$ & $94 \mathrm{Aa}$ & $100 \mathrm{Aa}$ \\
\hline 2 & $96 \mathrm{Aa}$ & $99 \mathrm{Aa}$ & 99 Aa & $100 \mathrm{Aa}$ & $100 \mathrm{Aa}$ & $100 \mathrm{Aa}$ & $94 \mathrm{Ba}$ & $91 \mathrm{Ba}$ & $97 \mathrm{Aa}$ & $88 \mathrm{Bb}$ \\
\hline 4 & $23 \mathrm{Db}$ & $80 \mathrm{Ab}$ & $58 \mathrm{Bb}$ & $75 \mathrm{Ab}$ & $30 \mathrm{Db}$ & $28 \mathrm{Db}$ & $28 \mathrm{Db}$ & $53 \mathrm{Bb}$ & $40 \mathrm{Cb}$ & $0 \mathrm{Ec}$ \\
\hline \multirow[t]{2}{*}{$\mathrm{CV}(\%)$} & 6.68 & & & & & & & & & \\
\hline & \multicolumn{9}{|c|}{ Índice de Velocidade de Germinação } & \\
\hline Recém-colhidas & $11,03 \mathrm{Ca}$ & $13,79 \mathrm{Ab}$ & $12,33 \mathrm{Ba}$ & $10,61 \mathrm{Ca}$ & $12,13 \mathrm{Bb}$ & $12,03 \mathrm{Ba}$ & $11,62 \mathrm{Ba}$ & $9,64 \mathrm{Ca}$ & $10,10 \mathrm{Ca}$ & $14,62 \mathrm{Aa}$ \\
\hline 2 & $7,16 \mathrm{Fb}$ & $17,18 \mathrm{Aa}$ & $11,25 \mathrm{Ca}$ & $9,54 \mathrm{Da}$ & $13,96 \mathrm{Ba}$ & $8,28 \mathrm{~Eb}$ & $7,43 \mathrm{Fb}$ & $6,52 \mathrm{Fb}$ & $6,85 \mathrm{Fb}$ & $4,96 \mathrm{~Gb}$ \\
\hline 4 & $1,04 \mathrm{Cc}$ & $5,66 \mathrm{Ac}$ & $3,51 \mathrm{Bb}$ & $4,01 \mathrm{Bb}$ & $1,53 \mathrm{Cc}$ & $1,25 \mathrm{Cc}$ & $1,23 \mathrm{Cc}$ & $2,52 \mathrm{Cc}$ & $1,71 \mathrm{Cc}$ & $0,00 \mathrm{Dc}$ \\
\hline \multirow[t]{2}{*}{$\mathrm{CV}(\%)$} & 10.25 & & & & & & & & & \\
\hline & \multicolumn{9}{|c|}{ Plântulas Normais Vigorosas (\%) } & \\
\hline Recém-colhidas & $87 \mathrm{Aa}$ & $84 \mathrm{Aa}$ & $72 \mathrm{Ba}$ & $78 \mathrm{Ba}$ & $93 \mathrm{Aa}$ & $77 \mathrm{Ba}$ & $78 \mathrm{Ba}$ & $56 \mathrm{Ca}$ & $78 \mathrm{Ba}$ & $94 \mathrm{Aa}$ \\
\hline 2 & $11 \mathrm{Cb}$ & $63 \mathrm{Ab}$ & $50 \mathrm{Ab}$ & $32 \mathrm{Bb}$ & $56 \mathrm{Ab}$ & $29 \mathrm{Bb}$ & $17 \mathrm{Cb}$ & $12 \mathrm{Cb}$ & $3 \mathrm{Cb}$ & $17 \mathrm{Cb}$ \\
\hline 4 & $0 \mathrm{Ac}$ & $2 \mathrm{Ac}$ & $0 \mathrm{Ac}$ & $0 \mathrm{Ac}$ & $0 \mathrm{Ac}$ & $0 \mathrm{Ac}$ & $0 \mathrm{Ac}$ & $0 \mathrm{Ac}$ & $0 \mathrm{Ab}$ & $0 \mathrm{Ac}$ \\
\hline \multirow[t]{2}{*}{ CV $(\%)$} & 20.61 & & & & & & & & & \\
\hline & \multicolumn{8}{|c|}{ Plântulas Normais Fracas (\%) } & & \\
\hline Recém-colhidas & $12 \mathrm{Cb}$ & $15 \mathrm{Cb}$ & $24 \mathrm{Bb}$ & $22 \mathrm{Bb}$ & $7 \mathrm{Cb}$ & $21 \mathrm{Bb}$ & $23 \mathrm{Bb}$ & $40 \mathrm{Ab}$ & $14 \mathrm{Cb}$ & $4 \mathrm{Cb}$ \\
\hline 2 & $85 \mathrm{Aa}$ & $32 \mathrm{Ea}$ & $48 \mathrm{Da}$ & $62 \mathrm{Ca}$ & $43 \mathrm{Da}$ & $70 \mathrm{Ba}$ & $76 \mathrm{Ba}$ & $77 \mathrm{Ba}$ & $91 \mathrm{Aa}$ & $75 \mathrm{Ba}$ \\
\hline 4 & $0 \mathrm{Ac}$ & $5 \mathrm{Ab}$ & $1 \mathrm{Ac}$ & $10 \mathrm{Ac}$ & $0 \mathrm{Ab}$ & $3 \mathrm{Ac}$ & $5 \mathrm{Ac}$ & $0 \mathrm{Ac}$ & $0 \mathrm{Ac}$ & $0 \mathrm{Ab}$ \\
\hline $\mathrm{CV}(\%)$ & 26.59 & & & & & & & & & \\
\hline
\end{tabular}

*Médias seguidas de mesma letra maiúsculas na linha comparam os genótipos em cada período de secagem e letras minúsculas na coluna comparam a resposta de cada genótipo entre os períodos de secagem, pelo teste de Scott-Knott a 0,05 de probabilidade. CV = Coeficiente de variação.

foi mais acentuada, variando entre 40 a $67 \%$. A composição química da semente também influencia diretamente na taxa de perda de umidade, sendo, portanto fundamental explorar esta característica considerando os genótipos de cacaueiro. Além disto, o tamanho da semente também pode ter influenciado na capacidade da estrutura de manter-se úmida ao longo da secagem, principalmente no caso do TSH-1188, cujas sementes são bem maiores que os demais genótipos (Venial et al. 2017).

O processo germinativo foi influenciado pela secagem das sementes, sendo a intensidade da resposta variável em função do genótipo (Tab. 1). A qualidade fisiológica das sementes recém-colhidas dos genótipos apresentou certa variação comparando com o ensaio de temperatura indicando haver diferenças entre os lotes estudados, possivelmente em função da época de colheita. No entanto, o genótipo TSH-1188, continuou sendo o mais vigoroso e foi o que apresentou menor sensibilidade a perda de umidade. O genótipo Catongo foi o mais sensível à secagem, apresentando a maior redução no vigor já aos dois dias de secagem e $0 \%$ de germinabilidade após quatro dias. Os resultados encontrados neste ensaio quanto ao processo germinativo também foram diferentes do observado por Venial et al. (2017) ao efetuarem a secagem por dois dias também em bancada, no qual verificaram que a germinabilidade foi decrescente no PS-1319, Ipiranga-01 e TSH-1188.

Os genótipos TSH-1188 e Ipiranga apresentaram incremento no vigor com a secagem aos dois dias, mesmo apresentando redução de $33 \%$ e $40 \%$, respectivamente, no teor de umidade. Os genótipos ESFIP-02 e CCN-51 mantiveram o vigor mesmo com a redução de cerca de $40 \%$ do teor de umidade inicial. Provavelmente o metabolismo germinativo não foi paralisado com a secagem aos dois dias e o desenvolvimento do eixo embrionário continuou mesmo com a redução na umidade. Esta perda de umidade provavelmente promoveu alterações fisiológicas nas sementes, contudo, principalmente os genótipos TSH-1188 e Ipiranga-01, devem ter apresentado alguns mecanismos de tolerância, que proporcionaram a protrusão radicular 
mais rápida nas sementes secas a $35 \%$ de umidade. No entanto, com a continuidade da secagem não foi suficiente para manter a viabilidade.

Assim como observado no ensaio de temperatura, o processo pós-germinativo foi o mais afetado com a redução da umidade das sementes, sendo observada menor porcentagem de plântulas normais vigorosas para todos os genótipos (Tab. 1). Os genótipos TSH-1188, ESFIP-02 e Ipiranga-01 apresentaram a maior porcentagem de plântulas normais vigorosas aos dois dias de secagem, seguido do CCN-51 e SJ-02. Este resultado confirma o que foi observado no processo germinativo, reforçando o indicativo da presença de alguns mecanismos de tolerância com as sementes secas entre $28 \%$ e $35 \%$ de umidade. Aos quatro dias, com redução entre $64 \%$ a $73 \%$ do teor de umidade, todos os genótipos apresentaram valores praticamente nulos de plântulas normais vigorosas e fracas.

$\mathrm{O}$ teste de agrupamento por meio dos componentes principais (Fig. 3), também foi eficiente na indicação de genótipos com comportamento distinto em relação à secagem. Nas sementes que foram secas durante dois dias pode-se observar a formação de grupos com níveis de sensibilidade diferenciada no agrupamento considerando o índice de velocidade de germinação e porcentagem de plântulas normais vigorosas (Fig. 3B). O TSH-1188 ficou totalmente isolado, enquanto o ESFIP-02 e Ipiranga-01 formaram um grupo com sensibilidade distinta dos demais. Pelo fato do TSH-1188 ser bastante vigoroso, a rápida germinação fez com que ele ficasse menos no teor intermediário de água, no qual ocorrem processos deletérios. Segundo Liang \& Sun (2002), a tolerância à dessecação das sementes do cacaueiro é melhorada quando elas ficam menos tempo no estado parcialmente seco.

As sementes do cacaueiro são recalcitrantes, no entanto, os teores de umidade crítico e letal variam entre os genótipos, sendo os resultados encontrados na literatura similares aos obtidos neste trabalho. Segundo Fajardo et al. (2011), estudando a sensibilidade a secagem nos genótipos UF667 e UF668 (Trinitario), Pound e EET 48 (Forasteros) e Carmelo (Criollo), concluíram que a sensibilidade a dessecação varia entre os genótipos das três origens genéticas a $30 \%$, contudo não toleram a secagem a $20 \%$. Com espécies do gênero Theobroma este comportamento também é similar, ficando os teores críticos entre $30 \%$ e o letal cerca de 15\% (Cruz \& Cicero 2008, Nascimento \& Carvalho 2012).
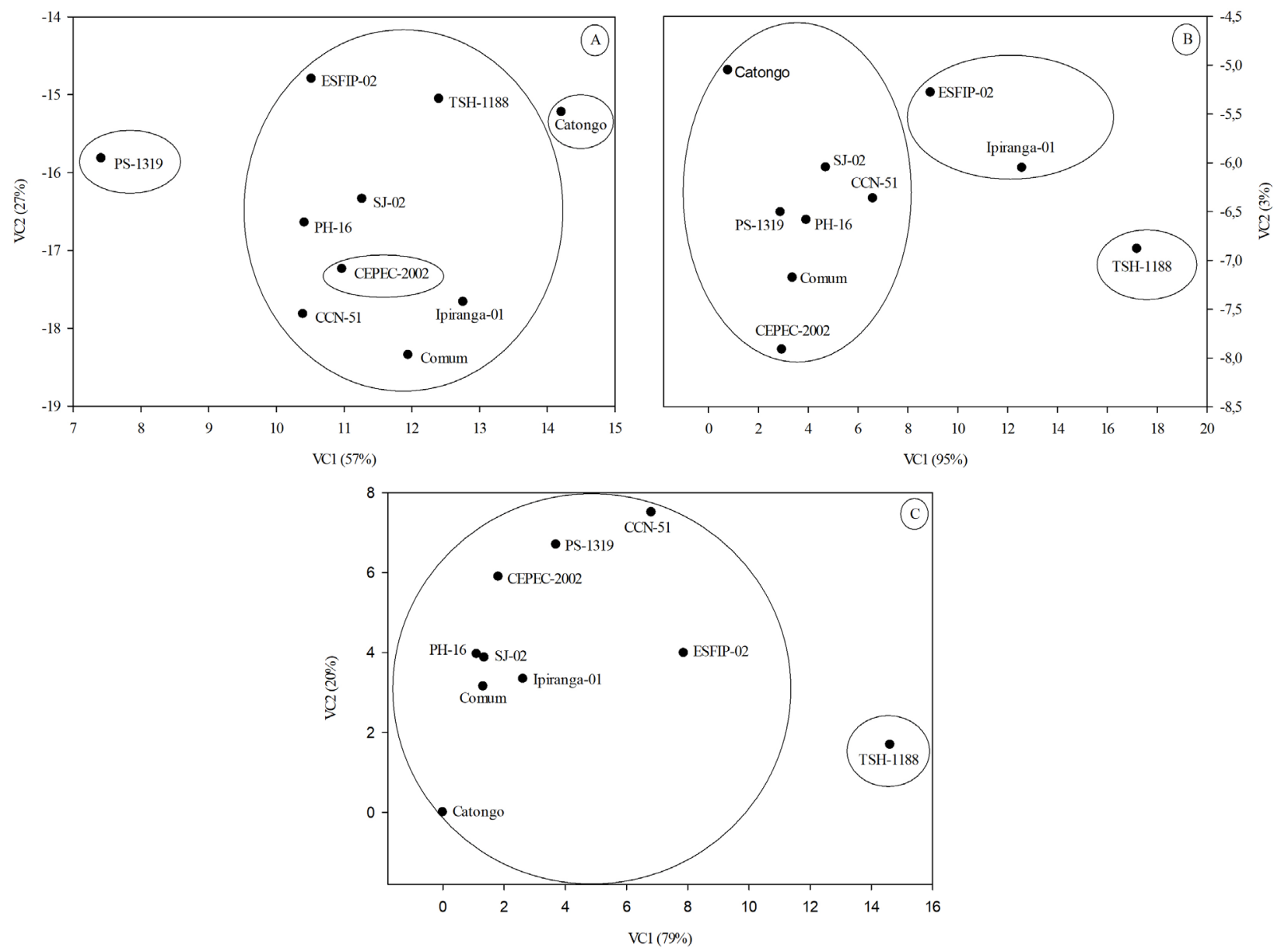

Fig. 3. Dispersão de dez genótipos de cacaueiro da Comissão Executiva do Plano da Lavoura Cacaueira no município de Linhares/ES, usando as coordenadas obtidas da matriz de distância generalizada de Mahalanobis $\left(\mathrm{D}^{2}\right)$, pelo procedimento estatístico baseado na minimização das diferenças entre as distâncias originais e aquelas no gráfico para o período de secagem de dois dias 
A sensibilidade à secagem das sementes do cacaueiro está muito relacionada com a diminuição da proteção enzimática contra os estresses oxidativos induzidos pela dessecação. Segundo Changrun \& Wendell (1999), abaixo do teor de umidade crítico as atividades das enzimas de degradação de radicais livres (ascorbato peroxidase, peroxidase e superóxido dismutase) diminuíram rapidamente, sendo observado acentuado aumento na peroxidação lipídica e extravasamento de eletrólitos.

Em sementes recalcitrantes, a redução do volume celular durante o processo de secagem promove um dano mecânico acentuado, ocorrendo uma acentuada redução de volume celular, podendo ocasionar a ruptura de plasmodesma nos três sistemas de tecidos fundamentais (dérmico, fundamental e vascular), fazendo com que o conteúdo citoplasmático seja lixiviado refletindo nos altos valores de condutividade elétrica (Silva et al. 2007). Chandel et al. (1995) analisaram eixos embrionários do cacaueiro submetidos a secagem até o nível crítico de umidade e observaram um dano geral na membrana, onde todas as inclusões ligadas à membrana, como núcleos, mitocôndrias, retículo endoplasmático e complexo de Golgi foram completamente interrompidas.

Em síntese, a resposta à temperatura foi influenciada de maneira distinta pelo genótipo, apresentando máxima germinabilidade entre $24^{\circ} \mathrm{C}$ a $32^{\circ} \mathrm{C}$, sendo a formação de plântulas severamente limitada pelos extremos térmicos avaliados $\left(15^{\circ} \mathrm{C}\right.$ e $\left.40^{\circ} \mathrm{C}\right)$.

O processo germinativo foi influenciado pela secagem das sementes, sendo a intensidade da resposta variável em função do genótipo. TSH-1188 pode ser considerado o genótipo com maior tolerância a secagem, enquanto o Catongo, o mais sensível.

\section{REFERÊNCIAS}

Afoakwa, E. O., Kongor, J. E., Takrama, J \& Budu, A. S. 2013. Changes in nib acidiFcation and biochemical composition during fermentation of pulp pre-conditioned cocoa (Theobroma cacao) beans. International Food Research Journal 20: 1843-1853.

Aime, M. C \& Phillips-Mora, W. 2005. The causal agents of witches'broom and frosty pod rot of cacao (chocolate, Theobroma cacao) form a new lineage of Marasmiaceae. Mycologia 97: 1012-1022.

Ali, M. K., Azhar, A \& Galani, S. 2013. Response of rice (Oryza sativa L.) under elevated temperature at early growth stage: physiological markers. Russian Journal of Agricultural and Socio-Economic Sciences 20:11-19.

Alvares, C. A., Stape, J. L., Sentelhas, P. C., Gonçalves, J. L. M \& Sparovek, G. 2013. Köppen's climate classification map for Brazil. Meteorologische Zeitschrift 22(6): 711-728. https://doi. org/10.1127/0941-2948/2013/0507

Amri, E. 2011. Germination of Terminalia sericea Buch. Ex DC seeds: The effects of temperature regime, photoperiod, gebberellic acid and potassium nitrate. International Journal of Applied Biology and Pharmaceutical Technology 2: 104-110. https://doi.org/10.1111/ j.1469-8137.2012.04086.x

Arévalo, E., Ram, A \&Valle, R. R. 2012. Integração de práticas de manejo no cultivo de cacaueiro. In Ciência, Tecnologia e manejo do cacaueiro (Valle, R. R. Valle ed.). Comissão Executiva de Planejamento da Lavoura Cacaueira (CEPLAC), Itabuna, p. 545-595.

Ataíde, G. M., Borges, E. E. L., Gonçalves, J. F. C., Guimarães, V. M \& Flores A. V. 2016. Alterações fisiológicas durante a hidratação de sementes de Dalbergia nigra ((Vell.) Fr. All. ex Benth.). Ciência Florestal 26: 615-625. http://dx.doi.org/10.5902/1980509822761

Brasil. Ministério da Agricultura, Pecuária e Abastecimento 2009. Regras para análise de sementes. Ministério da Agricultura, Pecuária e Abastecimento, Secretaria de Defesa Agropecuária, Brasília. 395p.

Bucheli, P., Rousseau, G., Alvarez, M., Laloi, M \& McCarthy, J. 2001. Developmental Variation of Sugars, Carboxylic Acids, Purine Alkaloids, Fatty Acids, and Endoproteinase Activity during Maturation of Theobroma cacao L. Seeds. Journal Agricultural and Food Chem 49: 5046-5051. http://dx.doi.org/ 10.1021/jf010620z

Comissão Executiva do Plano da Lavoura Cacaueira - CEPLAC. Lista de clones. Disponível em: http://www.ceplac.gov.br/radar/cacau. Acessado em 15.05.2017.

Chandel, K. P. S., Chaudhury, R., Radhamani, J \& Malik, S. K. 1995. Desiccation and Freezing Sensitivity in recalcitrante seeds of tea, cocoa and jackfruit. Annals of Botany Company 76: 443-450. https:// doi.org/10.1006/anbo.1995.1118

Changrun, L \& Wendell, Q. S. 1999. Desiccation sensitivity and activities of free radical-scavenging enzymes in recalcitrant Theobroma cacao seeds. Seed Science Research 9: 209-17. https://doi.org/10.1017/ S0960258599000215

Corrêa, F. L. O., Mendonça, V., Araújo, I. P., Vichiatto, M., Cidin, A. C. M \& Mendonça, L. F. M. 2011. Substratos e fertilizantes de liberação controlada na produção de mudas de cacaueiro (Theobroma cacao L.). Agrotrópica 23: 87-94.

Cruz, C. D. O 2006. Programa Genes - Análise multivariada e simulação. Editora Universidade Federal de Viçosa, Minas Gerais. 175 p.

Cruz, E. D \& Cicero, S. M. 2008. Sensitivity of seed to desiccation in cupuassu (Theobroma grandiflorum (Willd. ex Spreng.) K. Schum. - Sterculiaceae. Scientia Agricola 65: 557-560. http://dx.doi. org/10.1590/S0103-90162008000500017

Custódio, C. C., Vivan, M. R., Nunes, R. C. A \& Agostini, E. A. T. 2009. Tolerância cruzada induzida por choque térmico na germinação de sementes de feijão. Revista Brasileira de Sementes 31: 131-143. http://dx.doi.org/10.1590/S0101-31222009000100015

Dousseau, S., Alvarenga, A. A., Guimarães, R. M., Lara, T. S., Custódio, T. N \& Chaves, I. S. 2011. Ecofisiologia da germinação de sementes de Campomanesia pubescens. Ciência Rural 41:1362-1368. http:// www.redalyc.org/html/331/33119312005/

Dousseau, S., Alvarenga, A. A., Arantes. L. O., Izabel de Souza Chaves, I. S.\& Avelino, E. V. 2013. Techonolgy of Qualea grandiflora Mart. (Vochysiaceae) seeds. Cerne.19: 93-101. http://dx.doi.org/10.1590/ S0104-77602013000100012

Dürr, C., Dickie, J. B., Yang, X-Y \& Pritchard, H. W. 2015. Ranges of critical temperature and water potential values for the germination of species worldwide: Contribution to a seed trait database. Agricultural and Forest Meteorology 200: 222-232. https://doi.org/10.1016/j. agrformet.2014.09.024

Engelbrecht, C. J. B \& Harrington, T. C. 2005. Intersterility, morphology and taxonomy of Ceratocystis fimbriata on sweet potato, cacao and sycamore. Mycologia 97: 57-69. https://doi.org/10.1080/1557253 6.2006 .11832839

Fajardo, M. A. R., Tellez, L. C., Andrade, A. P. L., Alvarado, A. D., Mancera, H. A. Z \& Monter, Y. A. V. 2011. Tolerancia a la desecacion en semillas de tres orígenes geneticos de cacao (Theobroma cacao L.). Revista Fitotecnia Mexicana 34: 175-182. http://www.scielo. org. $\mathrm{mx} / \mathrm{pdf} / \mathrm{rfm} / \mathrm{v} 34 \mathrm{n} 3 / \mathrm{v} 34 \mathrm{n} 3 \mathrm{a} 8$.pdf

Food and Agriculture Organization of the United Nations - FAO. Disponível em: http://www.fao.org/faostat/en/\#data/QC. Acessado em 30.07.2017.

Ferraz, I. D. K., Albuquerque, M. C. F., Calvi, G. P \& Farias, D. L. 2012. Critérios morfológicos e temperatura para avaliação da germinação das sementes de cupuaçu. Revista Brasileira de Fruticultura 34: 905-914. http://dx.doi.org/10.1590/S0100-29452012000300033

Ferreira, D. F. 2014. Sisvar: a Guide for its Bootstrap procedures in multiple comparisons. Ciência e Agrotecnologia 38(2): 109-112. http://dx.doi.org/10.1590/S1413-70542014000200001

Figueiredo, S. F. L. 1986a. Conservação da viabilidade da semente de cacau. I. Cinquenta anos de estudos, uma revisão bibliográfica. Revista Theobroma 16(1): 17-29. 
Figueiredo, S.F.L. 1986b. Conservação da viabilidade da semente de cacau. II. Tipificação do fruto e descrição da semente e da germinação. Revista Theobroma 16(2): 75-88.

Figueiredo, S.F.L. 1986c. Conservação da viabilidade da semente de cacau. III. Solução de éteres celulósicos. Revista Theobroma 16(3): 115-126.

Flores, A.V., Borges, E.E.L., Guimarães, V. M., Gonçalves, J. F. C., Ataíde, G. M \& Barros, D. P. 2014. Atividade enzimática durante a germinação de sementes de Melanoxylon brauna Schott sob diferentes temperaturas. Cerne 20: 401-408. http://dx.doi.org/10.1590/01047 760201420031399

Gomes, A.R.S. \& Sodré, G.A. 2015. Conventional vegetative propagation. In Supplying new cocoa planting material to farmers: a review of propagation methodologies (B. Laliberte, M. End, A.R.S. Gomes, G.A. Sodré, M. Guiltinan, R. Lockwood \& S. Maximova, eds). Bioversity International, Rome, Italy, p.34-61.

Holanda, A. E. R., Filho, S. M \& Diogo, I. J. S. 2015. Influência da luz e da temperatura na germinação de sementes de sabiá (Mimosa caesalpiniifolia Benth. - Fabaceae). Gaia Scientia 9: 22-27. http:// www.periodicos.ufpb.br/index.php/gaia/article/view/18535/13191

Lamarca, E. V., Bonjovani, M. R., Faria, J. M. R \& Barbedo, C. J. 2013. Germinação em temperatura sub-ótima de embriões de Inga vera subsp. Affinis obtidos sob diferentes condições ambientais. Rodriguésia 64: 877-885.

Liang, Y \& Sun, W. Q. 2002. Rate of dehydration and cumulative desiccation stress interacted to modulate desiccation tolerance of recalcitrant cocoa and ginkgo embryonic tissues. Plant Physiology 128: 1323-1331. https://doi.org/10.1104/pp.010616

Lira, J. M. S., Lara, T. S., Rodrigues, A. C., Dousseau, S., Magalhães, M. M \& Alvarenga, A. A. 2015. Cross-tolerance mechanism induction in melon seeds by priming prior drying. Ciência e Agrotecnologia 39: 131-137. http://dx.doi.org/10.1590/S1413-70542015000200004

Lloh, A. C., Omatta, G., Ogbadu, G. H \& Onyenekwe, P. C. 2014. Effects of elevated temperature on seed germination and seedling growth on three cereal crops in Nigeria. Scientific Research and Essays 9: 806-813. https://doi.org/10.5897/SRE2014.5968

Long, R. L., Gorecki, M. J., Renton, M., Scott, J. K., Olville, L., Goggin, D. E., Commander, L. E., Westcott, D. A., Herry, H \& Finch-Savage, W. E. 2014. The ecophysiology of seed persistence: a mechanistic view of the journey to germination or demise. Biological Reviews 90: 31-59. https://doi.org/10.1111/brv.12095

Lopes, R. R \& Franke, L. B. 2011. Aspectos térmico-biológico da germinação de sementes de cornichão anual sob diferentes temperaturas. Revista Brasileira de Zootecnia 40: 2091-2096. http:// dx.doi.org/10.1590/S1516-35982011001000004

Madeira, N. R., Amaro, G. B., Melo, R. A. C., Ribeiro, C. S. C \& Reifschneider, F. J. B. 2016. Compatibilidade de portaenxertos para pimentão em cultivo protegid. Horticultura Brasileira 34: 470-474. http://dx.doi.org/10.1590/s0102-053620160404

Maguire, J. D. 1962. Speed of germination-aid in selection and evaluation for seedling emergence and vigour. Crop Science Madson 2: 176-177. http://dx.doi.org/10.2135/cropsci1962.0011183X000200020033x

Marcos-Filho, J. 2015. Seed vigor testing: an overview of the past, present and future perspective. Scientia Agricola 72: 363-374. http://dx.doi. org/10.1590/0103-9016-2015-0007
Martini, M. H \& Tavares, D. Q. 2005. Reservas das sementes de sete espécies de Theobroma: revisão. Revista Instituto Adolfo Lutz 64: 10-19.

Mertz, L. M., Henning, F. A., Soares, R. C., Baldiga, R. F., Peske, F. B \& Moraes, D. M. 2009. Alterações fisiológicas em sementes de arroz expostas ao frio na fase de germinação. Revista Brasileira de Sementes 31: 254-262. http://www.scielo.br/pdf/rbs/v31n2/v31n2a31.pdf.

Nascimento, W. M. D. O \& Carvalho, J. E. U. 2012. Sensibilidade de sementes de cupuí (Theobroma subincanum) à redução do grau de umidade e a exposição à baixa temperatura. Revista Brasileira de Fruticultura 34: 915-920. http://dx.doi.org/10.1590/S010029452012000300034

Parent, B \& Tardieu, F. 2012. Temperature responses of developmental processes have not been affected by breeding in different ecological areas for 17 crop species. New Phytologist 194: 760-774. https:// doi.org/10.1111/j.1469-8137.2012.04086.x

Penfield, S., Josse, E. M., Kannangara, R., Gilday, A. D., Halliday, K. J \& Graham, I. A. 2005. Cold and light control seed germination through the bhlh transcription factor spatula. Current Biology 15: 1998-2006. https://doi.org/10.1016/j.cub.2005.11.010

Pereira, J. E. S., Fortes, G. R. L \& Silva, J. B. 2001. Efeito da aplicação de baixa temperatura em plantas de macieira sobre o crescimento durante a aclimatização. Pesquisa Agropecuária Brasileira 36: 89-95. http://dx.doi.org/10.1590/S0100-204X2001000100011.

Rosental, L., Nonogaki, H \& Fait, A. 2014. Activation and regulation of primary metabolism during seed germination. Seed Science Research 24: 1-15. https://doi.org/10.1017/S0960258513000391

Seo, M., Nambara, E., Choi, G \& Yamaguchi, S. 2009. Interaction of light and hormone signals in germinating seeds. Plant Molecular Biology 69: 463-472. https://doi.org/10.1007/s11103-008-9429-y

Silva, P. A., Diniz, K. A., Oliveira, J. A \& Pinho, E. V. R. V. 2007. Análise fisiológica e ultra-estrutural durante o desenvolvimento e a secagem de sementes de soja. Revista Brasileira de Sementes 29: 15-22. http:// dx.doi.org/10.1590/S0101-31222007000200003

Singh, D. 1981. The relative importance of characteres affeting genetic divergence. Indian Journal of Genetic and Plant Breeding 41: 237-245.

Schmitz, J. D., Pasa, M. S., Fischer, D. L. O., Fachinello, J. C., Bianchi, V. J. 2014. Desempenho de porta-enxertos em diferentes sistemas de cultivo na produção de mudas do pessegueiro 'Chimarrita'. Revista Ceres 61: 155-160. http://dx.doi.org/ 10.1590/S0034$737 \mathrm{X} 2014000200020$

Socolowski, F., Vieira, D. C. M \& Takaki, M. 2008. Interaction of Temperature and Light on Seed Germination in Tecoma stans L. Juss. ex Kunth (Bignoniaceae). Brazilian Archives of Biology and Technology 51: 723-730. http://dx.doi.org/10.1590/s0102-053620160404

Toh, S., Imamura, A., Watanabe, A., Nakabayashi, K., Okamoto, M., Jikumaru, Y., Hanada, A., Aso, Y., Ishiyama, K., Tamura, N., Iuchi, S., Kobayashi, M., Yamaguchi, S., Kamiya, Y., Nambara, E \& Kawakami, N. 2008. High temperature-induced abscisic acid biosynthesis and its role in the inhibition of gibberellin action in arabidopsis seeds. Plant Physiology 146: 1368-1385. https://doi.org/10.1104/pp.107.113738

Venial, L. R., Alexandre, R. S., Camata, H., Lopes, J. C., Zanotti, R. F., Ferreira, A \& Aguilar, M. A. G. 2017. Biometria e armazenamento de sementes de genótipos de cacaueiro. Pesquisa Florestal Brasileira37:39-46. https://doi 10.4336/2017.pfb.37.89.1239 